Pacific Journal of Mathematics

GENERALIZED QUASICENTER AND HYPERQUASICENTER 


\title{
GENERALIZED QUASICENTER AND HYPERQUASICENTER OF A FINITE GROUP
}

\author{
J. B. DerR AND N. P. MuKherJeE
}

\begin{abstract}
The notion of quasicentral element is generalized to $p$ quasicentral element and the $p$-quasicenter and the $p$-hyperquasicenter are defined. It is shown that the $p$-quasicenter is $p$-supersolvable and the $p$-hyperquasicenter is $p$-solvable.
\end{abstract}

The quasicenter $Q(G)$ of a group $G$ is the subgroup of $G$ generated by all quasicentral elements of $G$, where an element $x$ of $G$ is called a quasicentral element ( $Q C$-element) when the cyclic subgroup $\langle x\rangle$ generated by $x$ satisfies $\langle x\rangle\langle y\rangle=\langle y\rangle\langle x\rangle$ for all elements $y$ of $G$. The hyperquasicenter $Q^{*}(G)$ of a group $G$ is the terminal member of the upper quasicentral series $1=Q_{0} \subset Q_{1} \subset Q_{2} \subset \cdots \subset Q_{n}=Q_{n+1}=Q^{*}(G)$ of $G$, where $Q_{i+1}$ is defined by $Q_{i+1} / Q_{i}=Q\left(G / Q_{i}\right)$. Mukherjee has shown [3,4] that the quasicenter of a group is nilpotent and the hyperquasicenter is the largest supersolvably immersed subgroup of a group. The proofs of these structure theorems rely on the fact that the powers of $Q C$-elements are again $Q C$-elements.

In this paper we generalize the notion of a quasicentral element in a way which allows the results about the quasicenter and the hyperquasicenter $[3,4]$ to be extended. All groups mentioned are assumed to be finite.

For a given group $G$ and a fixed prime $p$, the definition of $Q C$ element might suggest that an element $x$ of $G$ be called a $p$-quasicentral element provided $\langle x\rangle\langle y\rangle=\langle y\rangle\langle x\rangle$ holds for all $p$-elements $y$ of $G$. An apparent difficulty with this definition is that the powers of $p$-quasicentral elements need not again be $p$-quasicentral elements. For example, consider the group of order 18 defined by $G=\langle a, b$, $x\left|a^{3}=b^{3}=1=x^{2},[a, b]=1=[a, x],[b, x]=a\right\rangle$. A simple calculation shows that $a x$ is 3-quasicentral while $x=(a x)^{3}$ is not 3-quasicentralotherwise $\langle x\rangle\langle b\rangle=\langle b\rangle\langle x\rangle$ shall imply that $x$ normalizes $\langle b\rangle$, which is not the case however. Because of this example we choose to generalize the notion of a $Q C$-element as follows.

Definition 1. Let $G$ be a given group and $p$ a fixed prime. Suppose $x$ is an element of $G$ and let the order of $x$ be written as $|x|=p^{r} m$ where $(p, m)=1$. Then $x$ is called a $p$-quasicentral $(p-Q C)$ element of $G$ provided $\left\langle x^{m}\right\rangle\langle y\rangle=\langle y\rangle\left\langle x^{m}\right\rangle$ and $\left\langle x^{p^{r}}\right\rangle\langle y\rangle=\langle y\rangle\left\langle x^{p^{r}}\right\rangle$ hold for all $p$-elements $y$ of $G$. (It should be noted that every element of a $p^{\prime}$-group is $p$-QC.) 
THEOREM 1. If $x$ is a $p$-QC element of a group $G$ and $k$ is $a$ fixed integer, then $x^{k}$ is also a $p-Q C$ element of $G$.

Proof. Suppose $|x|=p^{b} m$ where $(p, m)=1$. Since $\left|x^{m}\right|=p^{b}$, $\left|x^{p^{b}}\right|=m$ and $x^{p^{b}}$ commutes with $x^{m},\langle x\rangle=\left\langle x^{p^{b}}\right\rangle\left\langle x^{m}\right\rangle=\left\langle x^{m}\right\rangle\left\langle x^{p^{b}}\right\rangle$. If $\left|x^{k}\right|=p^{c} n$ where $(p, c)=1$, then $\left(x^{k}\right)^{p^{c}}$ is a $p^{\prime}$-element of $\langle x\rangle$ and $\left(x^{k}\right)^{n}$ is a $p$-element of $\langle x\rangle$. It follows that $\left(x^{k}\right)^{p^{c}}$ is some power of $x^{p^{b}}$ and $\left(x^{k}\right)^{n}$ is some power of $x^{m}$. To show that $x^{k}$ is a $p$-QC element of $G$, it will suffice to show that $\left\langle\left(x^{m}\right)^{i}\right\rangle\langle y\rangle=\langle y\rangle\left\langle\left(x^{m}\right)^{i}\right\rangle$ and $\left\langle\left(x^{p^{b}}\right)^{i}\right\rangle\langle y\rangle=\langle y\rangle\left\langle\left(x^{p^{b}}\right)^{i}\right\rangle$ hold for all integers $i$ and all $p$-elements $y$ of $G$.

Let $y$ be any $p$-element in $G$. Since $x$ is a $p$-QC element of $G$, $\left\langle x^{m}\right\rangle\langle y\rangle=\langle y\rangle\left\langle x^{m}\right\rangle$. Therefore $\left\langle x^{m}\right\rangle\langle y\rangle$ is some subgroup $H$ of $G$ whose order divides $\left|x^{m}\right| \cdot|y|$. Since $x^{m}$ is then a $p$-QC element of the $p$ group $H, x^{m}$ is a $Q C$-element of $H$. It follows [3, 4] that every power of $x^{m}$ is a $Q C$-element of $H$. In particular, $\left\langle\left(x^{m}\right)^{i}\right\rangle\langle y\rangle=\langle y\rangle\left\langle\left(x^{m}\right)^{i}\right\rangle$ holds for every integer $i$.

Now proceed by induction on the order of $G$ to show that $\left\langle\left(x^{p^{b}}\right)^{i}\right\rangle\langle y\rangle=\langle y\rangle\left\langle\left(x^{p^{b}}\right)^{i}\right\rangle$ holds for every integer $i$ and every $p$-element $y$. Let $y$ be a fixed $p$-element of $G$ of order $p^{r}$. If $\left\langle x^{p^{b}}\right\rangle\langle y\rangle=\langle y\rangle\left\langle x^{p^{b}}\right\rangle$ is a proper subgroup of $G$, induction completes the argument. Assume therefore that $G=\left\langle x^{p^{b}}\right\rangle\langle y\rangle=\langle y\rangle\left\langle\left(x^{p^{b}}\right)\right\rangle$. Then $G$ is a supersolvable group (Theorem 13.3.1, [5]).

Let $\pi$ denote the set of prime divisors of $\left|x^{p^{b}}\right|=m$ which are larger than $p$. Since $G$ is supersolvable with order $|G|=p^{r} m, G$ has a normal Hall $\pi$-subgroup $K$. Distinguish two cases.

Case 1. $\pi$ is empty. Then $p$ is the largest prime dividing $|G|$. Since $\langle y\rangle$ is a Sylow $p$-subgroup of $G,\langle y\rangle$ must be normal in $G$. Clearly $\left\langle\left(x^{p^{b}}\right)^{i}\right\rangle\langle y\rangle=\langle y\rangle\left\langle\left(x^{p^{b}}\right)^{i}\right\rangle$ holds for all integers $i$ in this case.

Case 2. $\pi$ is nonempty. Let $s$ and $t$ denote integers such that $x_{1}=\left(x^{p^{b}}\right)^{s}$ is a $\pi$-element, $x_{2}=\left(x^{p^{b}}\right)^{t}$ is a $\pi^{\prime}$-element and $x^{p^{b}}=x_{1} x_{2}=$ $x_{2} x_{1}$ (Theorem 4, [2], p. 23). Then $\left\langle x_{1}\right\rangle$ is a Hall $\pi$-subgroup of $G$. Since $G$ is supersolvable, $\left\langle x_{1}\right\rangle \unlhd G$. It follows that $\left\langle x_{1}^{i}\right\rangle\langle y\rangle=\langle y\rangle\left\langle x_{1}^{i}\right\rangle$ holds for every integer $i$. Since $\left\langle\left(x^{p^{b}}\right)^{i}\right\rangle=\left\langle x_{1}^{i}\right\rangle\left\langle x_{2}^{i}\right\rangle$ for all integers $i$, the argument will be complete if we show $\left\langle x_{2}^{i}\right\rangle\langle y\rangle=\langle y\rangle\left\langle x_{2}^{i}\right\rangle$ holds for all $i$. Since $\left\langle x_{1}\right\rangle$ is a normal Hall $\pi$-subgroup of $G$, the SchurZassenhaus theorem shows that $G$ possesses a $\pi$-complement $R$. Since $y$ is a $\pi^{\prime}$-element of $G$, we may choose $R$ so that $y \in R$. Then $\langle y\rangle$ is a Sylow $p$-subgroup of $R$. Since $R$ is supersolvable and $p$ is the 
largest prime dividing $|R|,\langle y\rangle \unlhd R$. We now use the fact that $x_{2}$ is a $\pi^{\prime}$-element. Since $R$ is a Hall $\pi^{\prime}$ - subgroup of the solvable group $G$, some conjugate $x_{2}^{g}$ of $x_{2}$ lies in $R$. It now follows from $G=\left\langle x^{p^{b}}\right\rangle\langle y\rangle$ that $x_{2} \in R$, since every element $g$ in $G$ can be written as $\left(x^{p^{b}}\right)^{u} y^{v}$ for some integers $u, v$. Therefore $\left\langle x_{2}^{i}\right\rangle\langle y\rangle=\langle y\rangle\left\langle x_{2}^{i}\right\rangle$ holds for every integer $i$. This completes the proof of the theorem.

LEMMA 1. Let $\theta$ be a homomorphism from a group $G$ onto a group $\bar{G}$. If $x$ is a $p-Q C$ element of $G$, the image $x^{\theta}$ of $x$ is a $p-Q C$ element of $\bar{G}$.

Proof. Let $|x|=p^{b} m$ where $(p, m)=1$ and let $\left|x^{\theta}\right|=p^{c} n$ where $(p, n)=1$. It follows that $\langle x\rangle=\left\langle x^{p^{b}}\right\rangle\left\langle x^{m}\right\rangle$ and $\left\langle x^{\theta}\right\rangle=\left\langle\left(x^{\theta}\right)^{p^{c}}\right\rangle\left\langle\left(x^{\theta}\right)^{n}\right\rangle$. Now $\left\langle x^{\theta}\right\rangle=\langle x\rangle^{\theta}$ implies $\left\langle x^{p^{b}}\right\rangle^{\theta}=\left\langle\left(x^{\theta}\right)^{p^{c}}\right\rangle$ and $\left\langle x^{m}\right\rangle^{\theta}=\left\langle\left(x^{\theta}\right)^{n}\right\rangle$.

Let $\bar{u}$ be any $p$-element of $\bar{G}$. Then there is a $p$-element $y$ of $G$ with $y^{\theta}=\bar{u}$. Since $x$ is a $p-Q C$ element of $G,\left\langle x^{p^{b}}\right\rangle\langle y\rangle=\langle y\rangle\left\langle x^{p^{b}}\right\rangle$ and $\left\langle x^{m}\right\rangle\langle y\rangle=\langle y\rangle\left\langle x^{m}\right\rangle$. This shows $\left\langle x^{p^{b}}\right\rangle^{\theta}\langle y\rangle^{\theta}=\langle y\rangle^{\theta}\left\langle x^{p^{b}}\right\rangle^{\theta}$ and $\left\langle x^{m}\right\rangle^{\theta}\langle y\rangle^{\theta}=$ $\langle y\rangle^{\theta}\left\langle x^{m}\right\rangle^{\theta}$. Now $\langle y\rangle^{\theta}=\left\langle y^{\theta}\right\rangle=\langle\bar{u}\rangle$ implies $\left\langle\left(x^{\theta}\right)^{p^{0}}\right\rangle\langle\bar{u}\rangle=\langle\bar{u}\rangle\left\langle\left(x^{\theta}\right)^{p^{c}}\right\rangle$ and $\left\langle\left(x^{\theta}\right)^{n}\right\rangle\langle\bar{u}\rangle=\langle\bar{u}\rangle\left\langle\left(x^{\theta}\right)^{n}\right\rangle$. The proof of the lemma is therefore complete.

Definition 2. Let $G$ be a given group and $p$ a fixed prime. The $p$-quasicenter $Q_{p}(G)$ is the subgroup of $G$ generated by all $p-Q C$ elements of $G$.

We mention a few simple consequences of the definition of the $p$-quasicenter. For any group $G$ and any prime $p$, the quasicenter of $G$ is contained in the $p$-quasicenter of $G$. The $p$-quasicenter of a group is always a characteristic subgroup of the group. It should be noted that if a prime $p$ does not divide the order of a group $G$ then $Q_{p}(G)=G$.

TheOREM 2. For any group $G$ and every prime $p$, the $p$-quasicenter $Q_{p}(G)$ is p-supersolvable.

Proof. First we notice that $Q_{p}(G)=G$ is $p$-supersolvable if $p$ does not divide $|G|$. Consequently we assume that $p$ divides $|G|$. The proof is by induction on $|G|$.

It suffices to show that $G$ contains a nontrivial normal subgroup $N$ of order $p$ or of order prime to $p$. For, by induction, $Q_{p}(G / N)$ is then $p$-supersolvable. Since Lemma 1 shows $Q_{p}(G) N / N \subseteq Q_{p}(G / N)$ it will follow that $Q_{p}(G)$ is $p$-supersolvable. (This is because of the fact that normal subgroups of $p$-supersolvable groups are $p$-supersolvable and $N$ being of order $p$ or prime to $p$, the $p$-supersolvability of $Q_{p}(G) N / N$ implies $Q_{p}(G) N$ is $p$-supersolvable.) Since $Q_{p}\left(Q_{p}(G)\right)=Q_{p}(G)$, induction lets us assume that $Q_{p}(G)=G$. Thus $G$ is generated by $p-Q C$ 
elements $x_{1}, x_{2}, \cdots, x_{n}$. First we show that $G$ contains a proper normal subgroup. Distinguish two cases.

Case 1: Some $x_{i}$ has order divisible by $p$. Assume $p$ divides the order of $x_{1}$. Then there is an integer $d$ such that $\left|x_{1}^{d}\right|=p$. Since $x_{1}^{d}$ is a $p$-QC element of $G,\left\langle x_{1}^{d}\right\rangle$ permutes with each Sylow $p$-subgroup of $G$. Therefore $\left\langle x_{1}^{d}\right\rangle$ lies in the maximum normal $p$-subgroup $O_{p}(G)$ of $G$. Therefore $O_{p}(G)$ is a proper normal subgroup of $G$ or $O_{p}(G)=$ $G$ and $G$ is a $p$-group. If $G$ is a $p$-group, the theorem is trivially true.

Case 2: No $x_{i}$ has order divisible by $p$. Then $x_{1}, x_{2}, \cdots, x_{n}$ are $p-Q C$ elements of $G$ with $p^{\prime}$-orders. Since $|G|$ is divisible by $p, G$ must contain nonidentity $p$-elements. Let $T$ denote the subgroup of $G$ generated by all the $p$-elements of $G$. Since $T \unlhd G$, we can assume $T=G$. Therefore $G$ contains nonidentity $p$-elements $y_{1}, y_{2}, \cdots, y_{m}$ with $\left\langle y_{1}, y_{2}, \cdots, y_{m}\right\rangle=G$. Let $q$ be the largest prime dividing the product $\left|x_{1}\right| \cdot\left|x_{2}\right| \cdots\left|x_{n}\right|$. First suppose $p>q$. Since $x_{i}$ is a $p-Q C$ element and $y_{1}$ is a $p$-element, $\left\langle x_{i}\right\rangle\left\langle y_{1}\right\rangle=\left\langle y_{1}\right\rangle\left\langle x_{i}\right\rangle$ holds for all $i=1,2, \cdots, n$. It follows (theorem 13.3.1, [5]) that $\left\langle x_{i}\right\rangle\left\langle y_{1}\right\rangle$ is supersolvable of order $\left|x_{i}\right| \cdot\left|y_{1}\right|$ for $i=1,2, \cdots, n$. Since $x_{i}$ is a $p^{\prime}$-element and $p>q,\left\langle y_{1}\right\rangle$ is a normal Sylow $p$-subgroup of each group $\left\langle x_{1}\right\rangle\left\langle y_{1}\right\rangle$. Then $x_{1}, x_{2}, \cdots, x_{n}$ normalize $\left\langle y_{1}\right\rangle$ and $\left\langle y_{1}\right\rangle$ is a normal subgroup of $G=\left\langle x_{1}, x_{2}, \cdots, x_{n}\right\rangle$. Now suppose $p<q$ and let $\left|x_{1}\right|$ be divisible by $q$. Let $s$ be an integer such that $\left\langle x_{1}^{8}\right\rangle$ is a Sylow $q$-subgroup of $\left\langle x_{1}\right\rangle$. Since $\left\langle x_{1}\right\rangle\left\langle y_{j}\right\rangle=\left\langle y_{j}\right\rangle\left\langle x_{1}\right\rangle$ is a supersolvable group and $q$ is the largest prime dividing $\left|y_{j}\right| \cdot\left|x_{1}\right|, y_{j}$ normalizes $\left\langle x_{1}^{s}\right\rangle$ for $j=1,2, \cdots, m$. Therefore $\left\langle x_{1}^{s}\right\rangle \unlhd G=\left\langle y_{1}, y_{2}, \cdots, y_{m}\right\rangle$. This shows that in every case $G$ contains a proper normal subgroup $M$. If $M$ has order prime to $p$, we are finished. Assume now that $M$ is a minimal normal subgroup of $G$ and $p$ divides $|M|$. We will show that $|M|=p$.

Since $Q_{p}(G)=G, G$ is generated by $p$-QC elements $x_{1}, x_{2}, \cdots$, $x_{n}$ of $G$. For each $i, 1 \leqq i \leqq n,\left\langle x_{i}\right\rangle=\left\langle v_{1}\right\rangle\left\langle v_{2}\right\rangle \cdots\left\langle v_{d_{i}}\right\rangle$ where $v_{1}, v_{2}, \cdots, v_{d_{i}}$ are powers of $x_{i}, v_{1}$ is a $p$-element, and $v_{2}, v_{3}, \cdots, v_{d_{i}}$ are $p^{\prime}$-elements of prime power orders. Since powers of $p$-QC elements are also $p-Q C$ elements, it follows that $G$ can be written as $G=\left\langle a_{1}\right.$, $\left.a_{2}, \cdots, a_{h}, b_{1}, b_{2}, \cdots, b_{k}\right\rangle$ where each $a_{i}$ is a $p$-QC $p$-element of $G$ and each $b_{j}$ is a $p$-QC $p^{\prime}$-element of $G$ having prime power order.

Let $P$ denote the subgroup of $G$ generated by all $p$ - $Q C p$-elements of $G$. Clearly $P$ is a characteristic $p$-subgroup of $G$ with $\left\langle a_{1}, a_{2}, \cdots, a_{h}\right\rangle \subseteq P$. Since $M$ is a minimal normal subgroup of $G$, $P \cap M=1$ or $P \cap M=M$. First suppose that $P \cap M=1$. Then [P, $M] \leqq P \cap M=1$ and $P$ centralizes $M$. Let $w \in M$ with $|w|=p$. Clearly $a_{i}$ normalizes $\langle w\rangle$ for $i=1,2, \cdots, h$. Since each $b_{j}$ is a $p$-QC element of $G,\left\langle b_{j}\right\rangle\langle w\rangle=\langle w\rangle\left\langle b_{j}\right\rangle$ holds for $j=1,2, \cdots, k$. It follows that each group $\left\langle b_{j}\right\rangle\langle w\rangle$ is supersolvable of order $\left|b_{j}\right| \cdot|w|$. 
Since $\left|b_{j}\right|$ is a power of a prime other than $p,\left\langle b_{j}\right\rangle$ is a Sylow subgroup of $\left\langle b_{j}\right\rangle\langle w\rangle$. Hence $b_{j}$ normalizes $\langle w\rangle$ or $w$ normalizes $\left\langle b_{j}\right\rangle$ for each $j=1,2, \cdots, k$. Since $\left\langle b_{j}\right\rangle \cap M=1$ implies $b_{j}$ normalizes $\langle w\rangle$, $\langle w\rangle \unlhd G$ unless $\left\langle b_{j}\right\rangle \cap M \neq 1$ for some $j$. Assume that $\left\langle b_{d}\right\rangle \cap M \neq 1$ for some integer $d, 1 \leqq d \leqq k$. This implies that some prime different from $p$ divides the order of $M$. Since every power of $b_{d}$ is a $p-Q C$ element of $G, Q_{p}(M) \neq 1$. From the minimality of $M$ it follows that $Q_{p}(M)=M$, since $Q_{p}(M)$ is characteristic in $M$ and $M$ is normal in $G$. Induction applied to $M$ then shows that $M$ is $p$-supersolvable. If $N$ is a minimal normal subgroup of $M$ then $|N|$ is either $p$ or is prime to $p$. Then $T=\left\langle N^{g} \mid g \in G\right\rangle$ is a normal subgroup of $G$ contained in $M$ and $T=N^{g_{1}} \cdots N^{g}$ where $g_{1}, \cdots, g_{t}$ are elements of $G$. But $M$ being minimal normal in $G$ it follows that $T=M$. Therefore $M$ is either a $p$-group or a $p^{\prime}$-group, since $T$ is so. But $p$ divides the order of $M$ and therefore $M$ must be a $p$-group. This however contradicts the assumption that $\left\langle b_{d}\right\rangle \cap M \neq 1$. Thus $\langle w\rangle \unlhd G$. Since $\langle w\rangle \subseteq M, M=\langle w\rangle$ and $M$ has order $|w|=p$. Now suppose $P \cap M=M$. Then $M$ is a normal subgroup of the $p$-group $P$ and $M \cap Z(P) \neq 1$. Let $z$ be a nonidentity element of $M \cap Z(P)$ with $|z|=p$. Since $z \in Z(P)$, surely $\left\langle a_{1}, a_{2}, \cdots, a_{h}\right\rangle$ normalizes $\langle z\rangle$. On the other hand, $M$ being a $p$-group it is evident that $\left\langle b_{j}\right\rangle \cap M=1$ for each $j=1,2, \cdots, k$. As before, $\langle z\rangle \unlhd G$ unless $\left\langle b_{j}\right\rangle \cap M \neq 1$ for some $j$. Therefore $\langle z\rangle \unlhd G$. Since $1 \neq\langle z\rangle \subseteq M$, the minimality of $M$ shows $M=\langle z\rangle$. Therefore $M$ has order $|z|=p$ and the proof is complete.

Since the quasicenter of a group is nilpotent it is natural to ask if the $p$-quasicenter of a group must be $p$-nilpotent. We give an example to show that this need not be the case. Let $S_{3}$ denote the symmetric group of degree 3. The 3-quasicenter of $S_{3}$ is $S_{3}$ itself. Clearly $Q_{3}\left(S_{3}\right)=S_{3}$ is not 3-nilpotent.

Definition 3. Let $G$ be a given group and $p$ a fixed prime. The upper $p$-quasicentral series $1=H_{0} \subset H_{1} \subset \cdots \subset H_{n}=H_{n+1}$ of $G$ is the characteristic series where $H_{i+1}$ is defined by $H_{i+1} / H_{i}=Q_{p}\left(G / H_{i}\right)$. The number of distinct nontrivial terms in the upper $p$-quasicentral series of $G$ is called the $p$-quasicentral length of $G$. The terminal member of the upper $p$-quasicentral series of $G$ is called the $p$-hyperquasicenter of $G$. We denote this characteristic subgroup of $G$ by $Q_{p}^{*}(G)$.

THEOREM 3. In any group $G$, the p-hyperquasicenter $Q_{p}^{*}(G)$ is the intersection of all normal subgroups $N$ with $Q_{p}(G / N)=N / N$.

Proof. Let $S=\bigcap\left\{N \mid N \unlhd G\right.$ and $\left.Q_{p}(G / N)=N / N\right\}$. Clearly 
$S \subseteq Q_{p}^{*}(G)$. We now show that $Q_{p}^{*}(G)$ is included in every normal subgroup $N$ for which $Q_{p}(G / N)=N / N$. Let $1=H_{0} \subset H_{1} \subset H_{2} \subset \cdots \subset H_{m}=$ $Q_{p}^{*}(G)$ be the upper $p$-quasicentral series of $G$. Trivially $H_{0} \subseteq N$. Assume that $H_{i} \subseteq N$ and $H_{i+1} \varsubsetneqq N$. Then for some $p$-QC element $y H_{i}$ of $G / H_{i}, y \notin N$. This implies that under the natural homomorphism of $G / H_{i}$ to $G / N$, the $p-Q C$ element $y H_{i}$ is mapped onto the $p-Q C$ element $y N$ of $G / N$. Therefore $Q_{p}(G / N)$ is nontrivial, a contradiction. Hence $H_{i+1} \subseteq N$ and $Q_{p}^{*}(G) \subseteq N$ follows by induction.

We shall now investigate the structure of the $p$-hyperquasicenter $Q_{p}^{*}(G)$.

LEMma 2. Let $G$ be a group and $p$ a fixed prime. If $N \unlhd G$ and $N \subseteq Q_{p}^{*}(G)$ then $Q_{p}^{*}(G / N)=Q_{p}^{*}(G) / N$.

Proof. Let $1=H_{0} \subset H_{1} \subset H_{2} \subset \cdots \subset H_{n}=Q_{p}^{*}(G)$ be the upper $p$-quasicentral series of $G$ and let $N / N=L_{0} / N \subset L_{1} / N \subset \cdots \subset L_{k} / N=$ $Q_{p}^{*}(G / N)$ be the upper $p$-quasicentral series of $G / N$. By Lemma 1, $H_{1} N / N=Q_{p}(G) N / N \subseteq Q_{p}(G / N)=L_{1} / N$. Thus $H_{1} \subseteq L_{1} \subseteq L_{k}$. Now assume $H_{i} \subseteq L_{k}$ and deduce $H_{i+1} \subseteq L_{k}$. Since $H_{i} \subseteq L_{k}, G / L_{k}$ is a homomorphic image of $G / H_{i}$. Let $\theta$ be the natural homomorphism described by $\left(x H_{i}\right)^{\theta}=x L_{k}$. Then Lemma 1 shows that $\left(Q_{p}\left(G / H_{i}\right)\right)^{\theta} \subseteq$ $Q_{p}\left(G / L_{k}\right)=L_{k} / L_{k}$. Since $Q_{p}\left(G / H_{i}\right)=H_{i+1} / H_{i},\left(Q_{p}\left(G / H_{i}\right)\right)^{\theta}=H_{i+1} L_{k} / L_{k} \subseteq$ $L_{k} / L_{k}$. Therefore $H_{i+1} \subseteq L_{k}$ and by induction $H_{n} \subseteq L_{k}$. We complete, the proof by showing $L_{i} \subseteq H_{n}=Q_{p}^{*}(G)$ for each $i=1,2, \cdots, k$. By hypothesis $L_{0}=N \leqq Q_{p}(G)$. Now assume $L_{i} \leqq Q_{p}(G)$ and deduce $\mathrm{L}_{i+1} \subseteq$ $Q_{p}^{*}(G)$. Since $L_{i} \subseteq Q_{p}^{*}(G), G / Q_{p}^{*}(G)$ is a homomorphic image of $G / L_{i}$. The argument above can be repeated to obtain $L_{i+1} \subseteq Q_{p}^{*}(G)$.

THEOREM 4. For any group $G$ and any prime $p, Q_{p}^{*}(G)$ is $p$ solvable.

Proof. If $Q_{p}^{*}(G)=Q_{p}(G), Q_{p}^{*}(G)$ is $p$-supersolvable and the theorem is proved. Assume now that $Q_{p}(G) \varsubsetneqq Q_{p}^{*}(G)$. Let $N$ denote any minimal normal subgroup of $Q_{p}(G)$. Since $Q_{p}(G)$ is $p$-supersolvable, $N$ has $p^{\prime}$-order or $|N|=p$. Set $S=\left\langle N^{g} \mid g \in G\right\rangle$. Since $N \unlhd Q_{p}(G) \unlhd$ $G, N^{g} \unlhd Q_{p}(G)$ for each $\mathrm{g} \in G$. It follows that $S$ has order prime to $p$ or order a power of $p$. Since $S \unlhd G$ and $S \subseteq Q_{p}(G) \subseteq Q_{p}^{*}(G)$ induction shows that $Q_{p}^{*}(G / S)=Q_{p}^{*}(G) / S$ is $p$-solvable. Therefore $Q_{p}^{*}(G)$ is $p$ solvable.

It is possible to characterize the $p$-hyperquasicenter in terms of the normal subgroups included in it. We begin with the following definition.

Definition 4. Let $G$ be a group and $p$ a fixed prime. A normal subgroup $N$ of $G$ is called $p$-hyperquasicentral $(p-H Q)$ if $N / M \cap$ 
$Q_{p}^{*}(G / M) \neq M / M$ holds for each normal subgroup $M$ of $G$ which is properly contained in $N$.

The lemmas proved next will be useful for the proof of Theorem 5 .

Lemma 3. Let $G$ be any group and $p$ a fixed prime. If $N \unlhd G$ then $Q_{p}^{*}(G) N / N \subseteq Q_{p}^{*}(G / N)$.

Proof. Let $1=H_{0} \subset H_{1} \subset H_{2} \subset \cdots \subset H_{n}=Q_{p}^{*}(G)$ be the upper $p$ quasicentral series of $G$. By Lemma $1, H_{1} N / N=Q_{p}(G) N / N \subseteq Q_{p}(G / N) \subseteq$ $Q_{p}^{*}(G / N)=L / N$. Thus $H_{1} N \leqq L$. Now assume $H_{i} N \leqq L$ and deduce that $H_{i+1} N \subseteq L$. Since $H_{i} \subseteq H_{i} N, G / H_{i} N$ is a homomorphic image of $G / H_{i}$. Let $\phi$ be the natural homomorphism of $G / H_{i}$ onto $G / H_{i} N$ described by $\left(x H_{i}\right)^{\phi}=x H_{i} N$. Then Lemma 1 shows $\left(Q_{p}\left(G / H_{i}\right)\right)^{\phi} \subseteq$ $Q_{p}\left(G / H_{i} N\right)$. Since $Q_{p}\left(G / H_{i}\right)=H_{i+1} / H_{i}, H_{i+1} N / H_{i} N=\left(H_{i+1} / H_{i}\right)^{\phi} \subseteq$ $Q_{p}\left(G / H_{i} H\right)$. Next let $\theta$ be the natural homomorphism of $G / H_{i} N$ onto $G / L$ given by $\left(x H_{i} N\right)^{\theta}=x L$. By Lemma $1,\left(Q_{p}\left(G / H_{i} N\right)\right)^{\theta} \cong Q_{p}(G / L)=$ $L / L$. Since $H_{i+1} N / H_{i} N \subseteq Q_{p}\left(G / H_{i} N\right),\left(H_{i+1} N / H_{i} N\right)^{\theta}=H_{i+1} N L / L \subseteq$ $L / L$. Therefore $H_{i+1} N \leqq L$ and the assertion follows.

LEMMA 4. If any two groups $G_{1}$ and $G_{2}$ are isomorphic under a map $\theta$ then $\left(Q_{p}\left(G_{1}\right)\right)^{\theta}=Q_{p}\left(G_{2}\right)$.

Lemma 5. For any group $G$ and any prime $p$, the product of $p$-HQ subgroups of $G$ is a $p-H Q$ subgroup of $G$.

Proof. It suffices to show that for any $p-H Q$ subgroups $A$ and $B$ of $G$, the product $A B$ is a $p-H Q$ subgroup of $G$. Let $M$ be any normal subgroup of $G$ with $M \varsubsetneqq A B$. If $M \varsubsetneqq A$ or $M \varsubsetneqq B$ then $A B / M \cap Q_{p}^{*}(G / M) \neq M / M$. Now suppose $M$ is not a proper subgroup of either $A$ or $B$. Since $A \cap M=A$ and $B \cap M=B$ together imply $A B \subseteq M$, we may assume $R=A \cap M \subsetneq A$. Since $A$ is $p-H Q$, $A / R \cap Q_{p}^{*}(G / R) \neq R / R$. Let $y R$ be any nonidentity element of $A / R \cap$ $Q_{p}^{*}(G / R)$. Then $y \in A$ and $y \notin R$ show $y \notin M$. Since $M / R \unlhd G / R$, Lemma 3 shows $Q_{p}^{*}(G / R) \cdot M / R / M / R \subseteq Q_{p}^{*}(G / R / M / R)$. It now follows from the isomorphism of $G / R / M / R$ and $G / M$ that $y M$ is a nonidentity element of $Q_{p}^{*}(G / M)$. Therefore $A B / M \cap Q_{p}^{*}(G / M) \neq M / M$ and the assertion is proved.

Theorem 5. For any group $G$ and any prime $p, Q_{p}^{*}(G)$ is the product of all p-HQ subgroups of $G$.

Proof. Let $S$ denote the product of all $p-H Q$ subgroups of $G$. From Lemma 2 and the definition of $p-H Q$ subgroup it is easily seen that $Q_{p}^{*}(G)$ is a $p-H Q$ subgroup of $G$. Therefore $Q_{p}^{*}(G) \subseteq S$. 
Assume for the sake of contradiction that $Q_{p}^{*}(G) \varsubsetneqq S$. Since $S$ is a $p$ - $H Q$ subgroup of $G$ (Lemma 5) $S / Q_{p}^{*}(G) \cap Q_{p}^{*}\left(G / Q_{p}^{*}(G)\right) \neq Q_{p}^{*}(G) /$ $Q_{p}^{*}(G)$. Since $Q_{p}^{*}\left(G / Q_{p}^{*}(G)\right)=Q_{p}^{*}(G) / Q_{p}^{*}(G)$, this is the desired contradiction.

It should be remarked that for a set of primes $\pi, \pi$-quasicentrality can be defined in a manner analogous to $p$-quasicentrality. The $p$ quasicenter and $p$-hyperquasicenter can be extended in the natural way to obtain the notions of $\pi$-quasicenter and $\pi$-hyperquasicenter. It is easily checked that the results about the $p$-quasicenter and the $p$-hyperquasicenter of a group remain valid when $p$ is replaced by $\pi$.

\section{REFERENCES}

1. B. Huppert, Endliche Gruppen I, Springer-Verlag (1967).

2. W. Ledermann, Introduction to the Theory of Finite Groups, Interscience Publishers, Inc. (1964).

3. N. P. Mukherjee, The hyperquasicenter of a finite group I, Proc. Amer. Math. Soc., 26, No. 2 (1970), 239-243.

4. - The hyperquasicenter of a finite group I1, Proc. Amer. Math. Soc., 32, No. 1 (1972), 24-28.

5. W. R. Scott, Group Theory, Prentice Hall, Inc. (1964).

Received April 12, 1971 and in revised form January 13, 1972.

WeST VIRGINIA UNIVERSITY 


\section{PACIFIC JOURNAL OF MATHEMATICS}

\section{EDITORS}

\section{H. SAMELSON}

Stanford University

Stanford, California 94305

\section{R. HOBBY}

University of Washington

Seattle, Washington 98105

\section{J. DugundjI}

Department of Mathematics University of Southern California Los Angeles, California 90007

RICHARD ARENS

University of California

Los Angeles, California 90024

\section{ASSOCIATE EDITORS}
E. F. BECKENBACH
B. H. NeumanN
F. WOLF
K. YOSHIDA

\section{SUPPORTING INSTITUTIONS}

\author{
UNIVERSITY OF BRITISH COLUMBIA \\ CALIFORNIA INSTITUTE OF TECHNOLOGY \\ UNIVERSITY OF CALIFORNIA \\ MONTANA STATE UNIVERSITY \\ UNIVERSITY OF NEVADA \\ NEW MEXICO STATE UNIVERSITY \\ OREGON STATE UNIVERSITY \\ UNIVERSITY OF OREGON \\ OSAKA UNIVERSITY
}

\author{
UNIVERSITY OF SOUTHERN CALIFORNIA \\ STANFORD UNIVERSITY \\ UNIVERSITY OF TOKYO \\ UNIVERSITY OF UTAH \\ WASHINGTON STATE UNIVERSITY \\ UNIVERSITY OF WASHINGTON \\ AMERICAN MATHEMATICAL SOCIETY \\ NAVAL WEAPONS CENTER
}

The Supporting Institutions listed above contribute to the cost of publication of this Journal, but they are not owners or publishers and have no responsibility for its content or policies.

Mathematical papers intended for publication in the Pacific Journal of Mathematics should be in typed form or offset-reproduced, (not dittoed), double spaced with large margins. Underline Greek letters in red, German in green, and script in blue. The first paragraph or two must be capable of being used separately as a synopsis of the entire paper. The editorial "we" must not be used in the synopsis, and items of the bibliography should not be cited there unless absolutely necessary, in which case they must be identified by author and Journal, rather than by item number. Manuscripts, in duplicate if possible, may be sent to any one of the four editors. Please classify according to the scheme of Math. Rev. Index to Vol. 39. All other communications to the editors should be addressed to the managing editor, Richard Arens, University of California, Los Angeles, California, 90024.

50 reprints are provided free for each article; additional copies may be obtained at cost in multiples of 50 .

The Pacific Journal of Mathematics is published monthly. Effective with Volume 16 the price per volume (3 numbers) is $\$ 8.00$; single issues, $\$ 3.00$. Special price for current issues to individual faculty members of supporting institutions and to individual members of the American Mathematical Society: $\$ 4.00$ per volume; single issues $\$ 1.50$. Back numbers are available.

Subscriptions, orders for back numbers, and changes of address should be sent to Pacific Journal of Mathematics, 103 Highland Boulevard, Berkeley, California, 94708.

PUBLISHED BY PACIFIC JOURNAL OF MATHEMATICS, A NON-PROFIT CORPORATION

Printed at Kokusai Bunken Insatsusha (International Academic Printing Co., Ltd.), 270, 3-chome Totsuka-cho, Shinjuku-ku, Tokyo 160, Japan. 


\section{Pacific Journal of Mathematics}

\section{Vol. 42, No. $3 \quad$ March, 1972}

Catherine Bandle, Extensions of an inequality by Pólya and Schiffer for vibrating membranes ................................ 543

S. J. Bernau, Topologies on structure spaces of lattice groups.......... 557

Woodrow Wilson Bledsoe and Charles Edward Wilks, On Borel product measures .......................................

Eggert Briem and Murali Rao, Normpreserving extensions in subspaces of

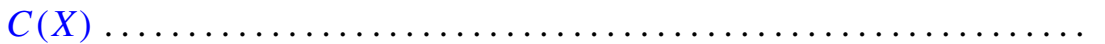

Alan Seymour Cover, Generalized continuation.................. 589

Larry Jean Cummings, Transformations of symmetric tensors .......... 603

Peter Michael Curran, Cohomology of finitely presented groups .......... 615

James B. Derr and N. P. Mukherjee, Generalized quasicenter and

hyperquasicenter of a finite group ...................... 621

Erik Maurice Ellentuck, Universal cosimple isols .................. 629

Benny Dan Evans, Boundary respecting maps of 3-mainfolds .......... 639

David F. Fraser, A probabilistic method for the rate of convergence to the

Dirichlet problem .................................. 657

Raymond Taylor Hoobler, Cohomology in the finite topology and Brauer

groups ..................................... 667

Louis Roberts Hunt, Locally holomorphic sets and the Levi form ........ 681

B. T. Y. Kwee, On absolute de la Vallée Poussin summability............ 689

Gérard Lallement, On nilpotency and residual finiteness in semigroups .... 693

George Edward Lang, Evaluation subgroups of factor spaces........... 701

Andy R. Magid, A separably closed ring with nonzero torsion pic ....... 711

Billy E. Rhoades, Commutants of some Hausdorff matrices ............. 715

Maxwell Alexander Rosenlicht, Canonical forms for local derivations . . . . 721

Cedric Felix Schubert, On a conjecture of L. B. Page ................ 733

Reinhard Schultz, Composition constructions on diffeomorphisms of $S^{p} \times S^{q}$

J. P. Singhal and H. M. (Hari Mohan) Srivastava, A class of bilateral generating functions for certain classical polynomials ....

Richard Alan Slocum, Using brick partitionings to establish conditions which insure that a Peano continuum is a 2-cell, a 2-sphere or an annulus...

James F. Smith, The p-classes of an $H^{*}$-algebra ...

Jack Williamson, Meromorphic functions with negative zeros and positive

poles and a theorem of Teichmuller ................. 